\title{
A multi-objective selective maintenance optimization method for series-parallel systems using NSGA-III and NSGA-II evolutionary algorithms
}

\author{
Xu, E.B. ${ }^{*}{ }^{\text {a }}$, Yang, M.S. ${ }^{\text {a }}$ Li, Y. ${ }^{\text {, }}$, Gao, X.Q. ${ }^{a}$, Wang, Z.Y. ${ }^{\text {a }}$, Ren, L.J. ${ }^{a}$

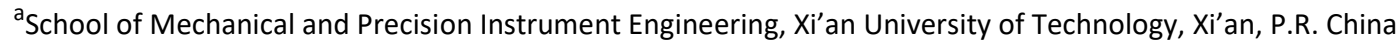

\begin{abstract}
A B S T R A C T
Aiming at the problem that the downtime is simply assumed to be constant and the limited resources are not considered in the current selective maintenance of the series-parallel system, a three-objective selective maintenance model for the series-parallel system is established to minimize the maintenance cost, maximize the probability of completing the next task and minimize the downtime. The maintenance decision-making model and personnel allocation model are combined to make decisions on the optimal length of each equipment's rest period, the equipment to be maintained during the rest period and the maintenance level. For the multi-objective model established, the NSGA-III algorithm is designed to solve the model. Comparing with the NSGA-II algorithm that only considers the first two objectives, it is verified that the designed multi-objective model can effectively reduce the downtime of the system.
\end{abstract}

\author{
ARTICLE INFO \\ Keywords: \\ Maintenance; \\ Series-parallel system; \\ Maintenance decision model; \\ Multi-objective optimization; \\ Selective maintenance; \\ Evolutionary algorithms; \\ Non-dominated sorting genetic \\ algorithm; \\ NSGA-II; \\ NSGA-III \\ *Corresponding author: \\ 1180211005@stu.xaut.edu.cn \\ (Xu, E.B.) \\ Article history: \\ Received 1 July 2021 \\ Revised 22 October 2021 \\ Accepted 25 October 2021
}

\section{(i)}

Content from this work may be used under the terms of the Creative Commons Attribution 4.0 International Licence (CC BY 4.0). Any further distribution of this work must maintain attribution to the author(s) and the title of the work, journal citation and DOI.

\section{References}

[1] Zhang, Y.P., Yang, K.X., Shi, L. (2018). Economic optimization model for imperfect preventive maintenance under reliability constraints, Computer Integrated Manufacturing Systems, Vol. 24, No. 12, 3019-3026, doi: 10.13196/ j.cims.2018.12.010.

[2] Laggoune, R., Chateauneuf, A., Aissani, D. (2010). Impact of few failure data on the opportunistic replacement policy for multi-component systems, Reliability Engineering \& System Safety, Vol. 95 No. 2, 108-119, doi: 10.1016/j.ress.2009.08.007.

[3] Rashidnejad, M., Ebrahimnejad, S., Safari, J. (2018). A bi-objective model of preventive maintenance planning in distributed systems considering vehicle routing problem, Computers \& Industrial Engineering, Vol. 120, 360-381, doi: 10.1016/j.cie.2018.05.001.

[4] Pandey, M., Zuo, M.J., Moghaddass, R., Tiwari, M.K. (2013). Selective maintenance for binary systems under imperfect repair, Reliability Engineering \& System Safety, Vol. 113, 42-51, doi: 10.1016/i.ress.2012.12.009. 
[5] Dao, C.D., Zuo, M.J., Pandey, M. (2014). Selective maintenance for multi-state series-parallel systems under economic dependence, Reliability Engineering \& System Safety, Vol. 121, No. 1, 240-249, doi: 10.1016/ j.ress.2013.09.003.

[6] Khatab, A., Aghezzaf, E.H., Diallo, C., Djelloul, I. (2017). Selective maintenance optimization for series-parallel systems alternating missions and scheduled breaks with stochastic durations, International Journal of Production Research, Vol. 55, No. 10, 3008-3024, doi: 10.1080/00207543.2017.1290295.

[7] Khatab, A. (2013). Hybrid hazard rate model for imperfect preventive maintenance of systems subject to random deterioration, Journal of Intelligent Manufacturing, Vol. 26, No. 3, 601-608, doi: 10.1007/s10845-013-0819-x.

[8] Chaabane, K., Khatab, A., Diallo, C., Aghezzaf, E.-H., Venkatadri, U. (2020). Integrated imperfect multimission selective maintenance and repairpersons assignment problem, Reliability Engineering \& System Safety, Vol. 199, Article No. 106895, doi: 10.1016/i.ress.2020.106895.

[9] Cheng, G., Zhou, B., Li, L. (2019). Joint optimization of production quantity, quality control and predictive maintenance for production systems with multiple machines, System Engineering - Theory \& Practice, Vol. 39, No. 8, 2152-2161, doi: 10.12011/1000-6788-2018-0104-10.

[10] Lu, Z., Zhao, C., Cui, W. (2017). Modeling and optimization of preventive maintenance scheduling for series production systems, Journal of Harbin Engineering University, Vol. 38, No. 2, 269-275, doi: 10.11990/jheu. 201511036.

[11] Leng, Q., Wang, L., Zhao, S., Zheng, Y. (2016). Integration and optimization of production batch and maintenance plan for multi-machine serial system, Computer Integrated Manufacturing System, Vol. 22, No. 8, 1945-1952, doi: 10.13196/i.cims.2016.08.013.

[12] Li, Y.T., Li, J., Huang, Z.K. (2019). Opportunistic maintenance strategy for serial-parallel manufacturing system under multiple-resource constraint, Journal of Lanzhou University of Technology, Vol. 45, No. 5, 33-38.

[13] Bousdekis, A., Papageorgiou, N., Magoutas, B., Apostolou, D., Mentzas, G. (2017). A proactive event-driven decision model for joint equipment predictive maintenance and spare parts inventory optimization, Procedia CIRP, Vol. 59, 184-189, doi: 10.1016/i.procir.2016.09.015.

[14] Lopes Gerum, P.C., Altay, A., Baykal-Gürsoy, M. (2019). Data-driven predictive maintenance scheduling policies for railways, Transportation Research Part C: Emerging Technologies, Vol. 107, 137-154, doi: 10.1016/i.trc.2019. $\underline{07.020}$.

[15] Xu, T.X., An, J., Zhang J.P., Li, Z.Q.(2018). Equipment condition maintenance decision-making model based on Markov approach, Journal of Gun Launch \& Control, Vol. 39, No. 3, 90-94, doi: 10.19323/j.issn.1673-6524.2018. $\underline{03.019}$.

[16] Pei, H., Hu, C.H., Si, X.S., Zhang, Z.X., Du, D.B. (2018). Equipment maintenance decision-making model based on remaining life prediction information under imperfect maintenance, Acta Automatica Sinica, Vol. 44, No. 4, 719729, doi: 10.16383/j.aas.2017.c160534.

[17] Nguyen, K.-A., Do, P., Grall, A. (2015). Multi-level predictive maintenance for multi-component systems, Reliability Engineering \& System Safety, Vol. 144, 83-94, doi: 10.1016/j.ress.2015.07.017.

[18] Dao, C.D., Zuo, M.J. (2017). Optimal selective maintenance for multi-state systems in variable loading conditions, Reliability Engineering \& System Safety, Vol. 166, 171-180, doi: 10.1016/i.ress.2016.11.006.

[19] Srinivas, N., Deb, K. (1994). Muiltiobjective optimization using nondominated sorting in genetic algorithms, Evolutionary Computation, Vol. 2, No. 3, 221-248, doi: 10.1162/evco.1994.2.3.221.

[20] Deb, K., Pratap, A., Agarwal, S., Meyarivan, T. (2002). A fast and elitist multiobjective genetic algorithm: NSGA-II, IEEE Transactions on Evolutionary Computation, Vol. 6, No. 2, 182-197, doi: 10.1109/4235.996017.

[21] Zhao, P.X., Luo, W.H., Han, X. (2019). Time-dependent and bi-objective vehicle routing problem with time windows, Advances in Production Engineering \& Management, Vol. 14, No. 2, 201-212, doi: 10.14743/ apem2019.2.322.

[22] Liu, Y.F., Zhang, Q.S. (2018). Solving multi-objective planning model for equipment manufacturing enterprises with dual uncertain demands using NSGA-II algorithm, Advances in Production Engineering \& Management, Vol. 13, No. 2, 193-205, doi: 10.14743/apem2018.2.284.

[23] Zhong, Y., Li, J.M., Zhu, S.Z. (2017). Research on the multi-objective optimized scheduling of the flexible job-shop considering multi-resource allocation, International Journal of Simulation Modelling, Vol. 16, No. 3, 517-526, doi: 10.2507/IJSIMM16(3)C013. 


\section{APEM}

Advances in Production Engineering \& Management Letnik 16 | Številka 3 | September 2021 | Strani 372-384 https://doi.org/10.14743/apem2021.3.407
ISSN 1854-6250

Spletna stran: apem-journal.org Izvirni znanstveni članek

\title{
Metoda večkriterijske optimizacije selektivnega vzdrževanja za zaporedno vzporedne sisteme z uporabo evolucijskih algoritmov NSGA-III in NSGA-II
}

\author{
Xu, E.B. ${ }^{*}$,, Yang, M.S. ${ }^{a}$, Li, Y. ${ }^{a}$, Gao, X.Q. ${ }^{a}$, Wang, Z.Y. ${ }^{a}$, Ren, L.J. ${ }^{a}$ \\ aschool of Mechanical and Precision Instrument Engineering, Xi'an University of Technology, Xi'an, P.R. China
}

\section{POVZETEK}

Trenutno se pri selektivnem vzdrževanju zaporedno vzporednih sistemov domneva, da so časi nedelovanja sistema konstantni in se omejeni viri ne upoštevajo. Da bi rešili ta problem, je predlagan model selektivnega vzdrževanja s tremi cilji: zmanjšanje stroškov vzdrževanja, povečanje verjetnosti dokončanja naslednje naloge in skrajšanje časa nedelovanja. Model odločanja o vzdrževanju in model razporeditve osebja sta združena za sprejemanje odločitev o optimalni dolžini neuporabe vsake opreme, o opremi, ki jo je treba vzdrževati v času neuporabe in o ravni vzdrževanja. Za reševanje postavljenega večkriterijskega modela je uporabljen algoritem NSGA-III. V primerjavi z algoritmom NSGA-II, ki upošteva le prva dva cilja, smo ugotovili, da lahko postavljen večkriterijski model učinkovito zmanjša čas nedelovanja sistema.

\author{
PODATKI O ČLANKU \\ Ključne besede: \\ Vzdrževanje; \\ Zaporedno vzporedni sistem; \\ Model odločanja o vzdrževanju; \\ Večkriterijska optimizacija; \\ Selektivno vzdrževanje; \\ Evolucijski algoritmi; \\ Genetski algoritem za nedominira- \\ no sortiranje; \\ NSGA-II; \\ NSGA-III \\ *Kontaktna oseba: \\ 1180211005@stu.xaut.edu.cn \\ (Xu, E.B.) \\ Zgodovina članka: \\ Prejet 1. julija 2021 \\ Popravljen 22. oktobra 2021 \\ Sprejet 25. oktobra 2021
}

\section{(i)}

Content from this work may be used under the terms of the Creative Commons Attribution 4.0 International Licence (CC BY 4.0). Any further distribution of this work must maintain attribution to the author(s) and the title of the work, journal citation and DOI. 